\title{
Exploring the $\mathrm{N} \alpha+3 \mathrm{n}$ light nuclei via the $\left({ }^{7} \mathrm{Li},{ }^{7} \mathrm{Be}\right)$ reaction
}

\author{
C. Nociforo ${ }^{1, a}$, F. Cappuzzello ${ }^{1}$, A. Cunsolo ${ }^{1,2}$, A. Foti ${ }^{2,3}$, S.E.A. Orrigo ${ }^{1}$, J.S. Winfield $^{1}$, M. Cavallaro ${ }^{1,2}$, S. Fortier ${ }^{4}$, \\ D. Beaumel ${ }^{4}$, and H. Lenske ${ }^{5}$ \\ 1 INFN-Laboratori Nazionali del Sud, Via S. Sofia 62, 95123 Catania, Italy \\ 2 Dipartimento di Fisica e Astronomia, Università di Catania, Via S. Sofia 64, 95123 Catania, Italy \\ 3 INFN-Sezione di Catania, Via S. Sofia 64, 95123 Catania, Italy \\ 4 Institut de Physique Nuclèaire, 91406 Orsay Cedex, France \\ ${ }^{5}$ Institut für Theoretische Physik, Universität Giessen, Heinrich-Buff-Ring 16, 35392 Giessen, Germany
}

Received: 18 June 2005 /

Published online: 29 March 2006 - (c) Società Italiana di Fisica / Springer-Verlag 2006

\begin{abstract}
Experimental signatures of the dynamical correlations of a core with a single-particle neutron have been found in light neutron-rich nuclei investigated via the $\left({ }^{7} \mathrm{Li},{ }^{7} \mathrm{Be}\right)$ charge-exchange reactions at around $8 \mathrm{MeV} / u$. Of particular astrophysical relevance are low-lying narrow resonances $(\Gamma \sim 200 \mathrm{keV}$ FWHM) BSEC (Bound States Embedded in the Continuum). Because of their long lifetime BSEC states are likely to effect the capture rates in any scenario for nucleosynthesis in neutron-rich environment. They have been observed in the continuum of ${ }^{11} \mathrm{Be}$ and ${ }^{15} \mathrm{C}$ nuclei. A microscopic nuclear structure model based on QRPA theory, which takes into account Dynamical Core Polarisation (DCP) correlations, gives a suitable description of these resonances as well as single-particle states of the studied systems. In this context, high-energy narrow structures populated in nuclei having an integer number of $\alpha$-particles plus three neutrons are good BSEC candidates and can be systematically investigated.
\end{abstract}

PACS. 21.10.Pc Single-particle levels and strength functions - 21.60.-n Nuclear structure models and methods - 25.70.Kk Charge-exchange reactions - 27.20.+n $6 \leq A \leq 19$

\section{Introduction}

During the last decade the investigation of the structure of unstable nuclei has become the major activity of nuclear structure physics, leading to the discovery of a variety of new phenomena, from halos and neutron skins to shell quenching and the appearances of new magic numbers. From a theoretical point of view the explanation of new phenomena represents a precious benchmark strongly supporting the development of refined theories based on the microscopic description of nuclei. Also, the studies of these phenomena have provided an interesting cross-relation to astrophysics because such investigations are most suitable for exploring under laboratory conditions reactions which otherwise occur only in stellar and supernovae environments. Their precise understanding is of importance to comprehend nucleosynthesis in both stellar and primordial processes.

The nuclei considered in this paper are located in the mass region of the $\mathrm{CNO}$ cycle. Although the nuclei investigated here are not directly involved in the $\mathrm{CNO}$ cycle, we

\footnotetext{
a Present address: GSI, Darmstadt, Germany; e-mail: c.nociforo@gsi.de
}

hope to bring attention to a general aspect of nuclear continuum dynamics, namely the existence of extremely sharp resonances in the low-energy continuum. These Bound States Embedded into the Continuum (BSEC) are longlived states resulting from configuration mixing between simple single-particle states attached to the (inert) ground state of the host system and more complex core-excited configurations which can only decay via the coupling to a continuum of unbound states attached to the core ground state.

Far from the line of $\beta$-stability the standard representation of nuclei, based on the assumption of mean-field dynamics, turns out to be often inadequate. The reason is the energy scale of the single-particle states given by the separation energy $S_{n}$, which is reduced to a few hundred $\mathrm{keV}$, value close to the typical matrix element of the residual nuclear interaction. Hence, in loosely bound systems a strong competition of mean-field and correlation dynamics is expected even at low excitation energy. In the case of ${ }^{11} \mathrm{Be}$ nucleus, for instance, this is supported by the observations of a considerable amount of ${ }^{10} \mathrm{Be}\left(2^{+}\right)$core excited configuration in the ground state, which was explored via transfer [1] as well as breakup reactions [2]. 
Experimental signatures of the Dynamical Core Polarisation (DCP) correlations above particle threshold are the BSEC [3,4], which were observed in the inelastic scattering of stable nuclei. The BSEC are quasibound resonant states characterized by a peculiar line shape. They are interpreted as configurations where a nucleon is re-scattered by a core, which can be easily excited into a multi-particle configuration with total energy above particle threshold but neither of the involved particles by itself is in a continuum state. Since such a core excited configuration is degenerate with the single particle continuum attached to the ground-state the decay will take place only through the relatively weak residual interaction. Hence, a nucleon trapped in such a configuration remains for long time attached to the core although the total energy of the direct process would be enough to remove it immediately.

Spectroscopic studies dealing with the weakly bound ${ }^{11} \mathrm{Be}$ and ${ }^{15} \mathrm{C}$ nuclei, explored via the $\left({ }^{7} \mathrm{Li},{ }^{7} \mathrm{Be}\right)$ chargeexchange (CEX) reactions at around $8 \mathrm{MeV} / u$, have shown the appearance of narrow resonances $(\Gamma \sim 200 \mathrm{keV}$ FWHM) far from the neutron emission threshold, identified most likely as BSEC $[5,6]$. In sect. 2 the experimental results are summarized. As pointed out in refs. [5,6], the coupling between the valence neutron and the excited states of the core is needed in order to get a correct description also of the observed continuum shape. For this reason, a microscopic nuclear structure model based on the QRPA theory, called DCP model $[7,8,9]$ has been applied to the studied nuclear systems. A brief review of it is given in sect. 3 . The obtained results are then discussed and compared to the experimental data.

Nuclei having a $N \alpha+3 n$ like configuration (where $N$ is an integer number) are good candidates to observe BSEC (see sect. 4). Clear indications may be provided by highresolution studies of the continuum structures for such nuclear systems. In the low-mass region experimental data obtained via the $\left({ }^{7} \mathrm{Li},{ }^{7} \mathrm{Be}\right) \mathrm{CEX}$ reaction at $\sim 8 \mathrm{MeV} / u$ are already available for the ${ }^{7} \mathrm{He}$ and ${ }^{19} \mathrm{O}$ nuclei, thus giving a systematic overview of these phenomena as a function of asymmetry and mass. Some conclusions are discussed in sect. 5 .

\section{The $\left({ }^{7} \mathrm{Li},{ }^{7} \mathrm{Be}\right) \mathrm{CEX}$ reaction}

The $\left({ }^{7} \mathrm{Li},{ }^{7} \mathrm{Be}\right)$ CEX reaction has been extensively studied in the past mainly because it gives useful information on the response function of nuclei to the isovector component of the effective nucleon-nucleon interaction $[10$, 11]. Since such reactions transform a target proton into a neutron, they have a natural relation to $\beta$ decay processes. Indeed, charge exchange reactions allow to study, under well-defined conditions, transitions of $\beta$ decay-type in mass regions which would be otherwise inaccessible. Such data are of direct relevance also for astrophysical purposes, especially for understanding weak interactions in short-lived neutron-rich nuclei far off stability. It is also known to be a useful spectroscopic tool at intermediate energies [12] where the one-step direct mechanism is considered to be dominant. In addition, depending on the system chosen and the $q$-matching condition [13], this reaction is an interesting spectroscopic probe at Tandem energies as well $[14,15,16,17]$. In ref. [17] the competition between the direct exchange of isospin degree of freedom and the two-step transfer mechanisms was faced for the ${ }^{11} \mathrm{~B}\left({ }^{7} \mathrm{Li},{ }^{7} \mathrm{Be}\right){ }^{11} \mathrm{Be}$ reaction. Due to the high selectivity of the $\left({ }^{7} \mathrm{Li},{ }^{7} \mathrm{Be}\right)$ reaction, the analogy between the $\beta$-decay probabilities and the CEX cross sections measured at forward angles for Gamow-Teller transitions, proved for the $(\mathrm{p}, \mathrm{n})$ or $(\mathrm{n}, \mathrm{p})$ reactions $[18,19]$, is maintained (within $20 \%$ accuracy) despite the complications coming from the presence of the heavy ions involved in the reaction [17]. In the case where the $\left({ }^{7} \mathrm{Li},{ }^{7} \mathrm{Be}_{\text {g.s. }}\right)$ and the $\left({ }^{7} \mathrm{Li},{ }^{7} \mathrm{Be}_{0.43}\right)$ transitions are experimentally resolved (by means of high resolution or $\gamma$-ray coincidence measurements), the observable $G=\sigma_{1} /\left(\sigma_{0}+\sigma_{1}\right)$, where $\sigma_{0}$ and $\sigma_{1}$ are the corresponding cross sections measured for the two cited transitions, gives a strong indication on how the reaction mechanism proceeds $[10,11]$. As a consequence of such an analysis, it seems that in the $\left({ }^{7} \mathrm{Li},{ }^{7} \mathrm{Be}\right)$ reaction involving ${ }^{11} \mathrm{Be}$ or ${ }^{15} \mathrm{C}$ the two-step components are hidden also due to the structure properties of these unstable nuclei.

\subsection{Experimental results}

During the last few years, several light stable and unstable nuclei, the latter having a $N \alpha+3 \mathrm{n}$ like configuration (where $N$ is an integer number) have been investigated with the same probe $\left({ }^{7} \mathrm{Li},{ }^{7} \mathrm{Be}\right)$ in a systematic way. All the experiments have been performed with a ${ }^{7} \mathrm{Li}^{+++}$ beam at around $8 \mathrm{MeV} / u$ provided by the Tandem Van de Graaff accelerator at the IPN-Orsay with different targets ( $\sim 100 \mu \mathrm{g} / \mathrm{cm}^{2}$ thickness), both solid and gas ones. In all the cases, the ${ }^{7}$ Be ejectiles were detected in the focal plane detector of the Split-Pole magnetic spectrometer with a resolution as good as $50 \mathrm{keV}$. The focal plane detector was a position- and angle-sensitive proportional gas counter followed by a stopping plastic scintillator. Measurements at forward angles including $0^{\circ}$ were done to get angular distribution of the cross sections. A $\Delta E-E$ telescope of silicon detectors was mounted at around $\theta_{l a b}=30^{\circ}$ and used as a monitor for normalization procedures. Additional runs with ${ }^{12} \mathrm{C}$ target such as other elements normally present as target impurities (or in the windows of the gas target) were performed in order to estimate the source of background in the spectra. The background due to the $\mathrm{p}\left({ }^{7} \mathrm{Li},{ }^{7} \mathrm{Be}\right) \mathrm{n}$ reaction was always present below $\theta_{\text {lab }}=7^{\circ}$ due to the large cross section $(\sim 100 \mathrm{mb} / \mathrm{sr})$.

Some interesting results have been obtained in the case of ${ }^{11} \mathrm{Be}\left(S_{n}=0.504 \mathrm{MeV}\right)$ and ${ }^{15} \mathrm{C}\left(S_{n}=1.218 \mathrm{MeV}\right)$ nuclei. Examples of final spectra detected in the case of ${ }^{11} \mathrm{~B}\left({ }^{7} \mathrm{Li},{ }^{7} \mathrm{Be}\right){ }^{11} \mathrm{Be}$ and ${ }^{15} \mathrm{~N}\left({ }^{7} \mathrm{Li},{ }^{7} \mathrm{Be}\right){ }^{15} \mathrm{C}$ reactions at around $8 \mathrm{MeV} / u$ are shown in figs. 1 and 2 , at $\theta_{l a b}=10.5^{\circ}$ and $\theta_{l a b}=14^{\circ}$, respectively. The experimental results show that the response of the two nuclei to the same probe is quite similar. Single-particle states are populated at low excitation energy $(\sim 100 \mu \mathrm{b} / \mathrm{sr})$. At high excitation energy, where the DCP regime is expected to be dominant, BSEC have been identified. In particular, in the ${ }^{11} \mathrm{Be}$ 


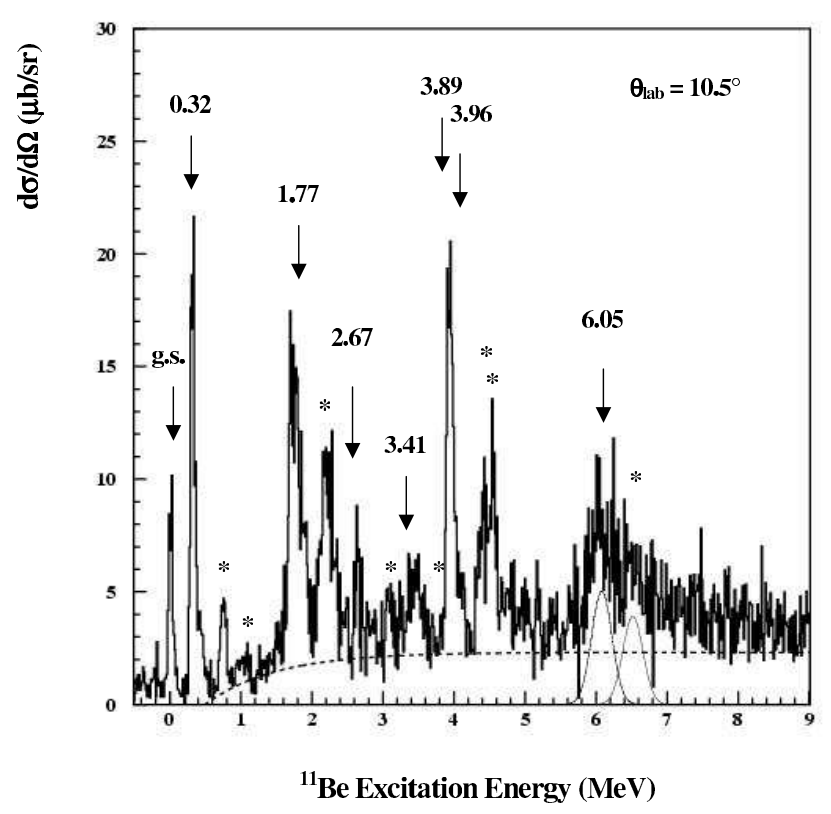

Fig. 1. Final excitation energy spectrum at $\theta_{l a b}=10.5^{\circ}$ for the ${ }^{11} \mathrm{~B}\left({ }^{7} \mathrm{Li},{ }^{7} \mathrm{Be}\right){ }^{11} \mathrm{Be}$ reaction at around $8 \mathrm{MeV} / u$. The fitted structure represents the state at $6.05 \mathrm{MeV}$. The peaks marked with an asterisk are associated to the excitation of ${ }^{7} \mathrm{Be}$ at $0.43 \mathrm{MeV}$. The dashed line is the non-resonant ${ }^{11} \mathrm{~B}\left({ }^{7} \mathrm{Li},{ }^{7} \mathrm{Be}\right){ }^{10} \mathrm{Be}+\mathrm{n}$ three-body phase space.

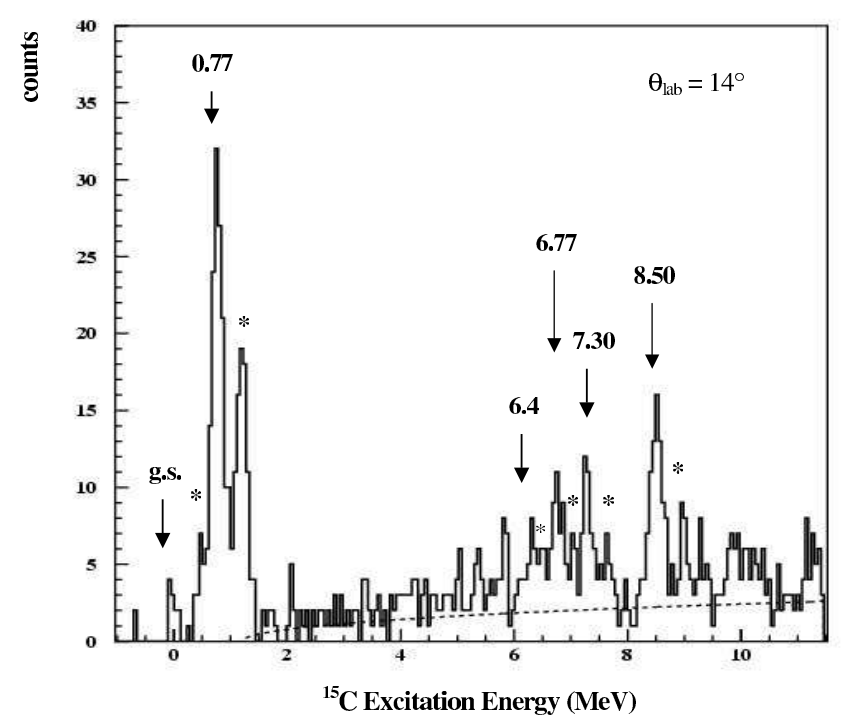

Fig. 2. Final excitation energy spectrum at $\theta_{l a b}=14^{\circ}$ for the ${ }^{15} \mathrm{~N}\left({ }^{7} \mathrm{Li},{ }^{7} \mathrm{Be}\right){ }^{15} \mathrm{C}$ reaction at around $8 \mathrm{MeV} / u$. The peaks indicated with arrows are the populated ones, those marked with an asterisk are associated to the excitation of ${ }^{7} \mathrm{Be}$ at $0.43 \mathrm{MeV}$. The dashed line is the non-resonant ${ }^{15} \mathrm{~N}\left({ }^{7} \mathrm{Li},{ }^{7} \mathrm{Be}\right){ }^{14} \mathrm{C}+\mathrm{n}$ three-body phase space.

spectrum the structure at $6.05 \mathrm{MeV}(\Gamma=320 \pm 40 \mathrm{keV})$ and in the ${ }^{15} \mathrm{C}$ spectrum the narrow peak at $8.50 \mathrm{MeV}$ $(\Gamma \leq 140 \mathrm{keV})$. Peaks marked by an asterisk refer to transitions in which the ${ }^{7} \mathrm{Be}$ is emitted in the $0.43 \mathrm{MeV}$ first (bound) excited state, and are well resolved due to the high resolution given by the Tandem beam and magnetic spectrometer combination. A non-resonant threebody phase space due to the projectile breakup was calculated analytically for the exit channel like in ref. [20], transformed in the proper excitation energy scale and normalised for the most backward angle measurement $\left(\theta_{l a b}=18^{\circ}\right)$. In both spectra it is plotted. The ${ }^{11} \mathrm{Be}$ state at $6.05 \mathrm{MeV}$ was previously unknown. For the ${ }^{15} \mathrm{C}$ case, narrow structures at around $7-8 \mathrm{MeV}$ were observed before in the less selective ${ }^{9} \mathrm{Be}\left({ }^{7} \mathrm{Li}, \mathrm{p}\right){ }^{15} \mathrm{C}$ reaction performed at $20 \mathrm{MeV}$ [21]. There, the attempts of spin assignment for these states led to high spin values, to account for the observed hindrance for neutron emission.

Our data were analyzed in a QRPA many-body approach using the formalism of ref. [22]. Special features of these exotic systems that must be taken into account are described in details in refs. $[5,17]$. Calculations of microscopic QRPA transition densities, assuming configurations where the valence neutron is coupled to an inert core, reproduce well in both cases the level structure below $2 \mathrm{MeV}[5,6]$, but are not able to explain the strong fragmentation of the strength at higher excitation energy.

A comparison with DWBA calculations, which was possible only for the ${ }^{11} \mathrm{Be}\left(\right.$ g.s., $\left.1 / 2^{+}\right),{ }^{11} \mathrm{Be}\left(0.32,1 / 2^{-}\right)$ and ${ }^{11} \mathrm{Be}\left(1.77,5 / 2^{+}\right)$states [17], and for the ${ }^{15} \mathrm{C}\left(\right.$ g.s., $\left.1 / 2^{+}\right)$and ${ }^{15} \mathrm{C}\left(0.77,5 / 2^{+}\right)$ones [23], shows that the corresponding angular distributions are quantitatively well reproduced without any scaling factor and without the necessity to introduce two-steps contributions. The unnatural-parity state transitions, both in the projectile and in the target, account for most of the observed cross sections. The distribution at around $0^{\circ}$ of the $G$ ratio as a function of the excitation energies are also well described in the DWBA framework.

In addition, a quite asymmetric line shape has been observed in the spectra of ${ }^{15} \mathrm{C}$ at several angles in correspondence of the $8.5 \mathrm{MeV}$ resonance (see fig. 2), explained as the result of the interference between the BSEC and the three-body continuum $[24,25]$. Such an observation is an important experimental signature of the existence of BSEC.

\section{Nuclear structure model}

Single-particle configurations of the odd-mass nuclei with respect to an even-even vibrating core nucleus have been investigated theoretically [26]. In ref. [27], correlations in the ${ }^{11} \mathrm{Be}$ nucleus were calculated as corrections to the Hartree-Fock potential due to the coupling of singleparticle states to RPA collective one-phonon states of the ${ }^{10} \mathrm{Be}$ core. The result, in case of the ${ }^{11} \mathrm{Be}$ ground state, leads to a strong admixture $(\sim 20 \%)$ of a $d_{5 / 2}$ neutron coupled to the ${ }^{10} \mathrm{Be}\left(2^{+}\right)$core, in agreement with experimental results (see [2] for a review). In these calculations a non microscopic effective interaction was used. Recently, coupling of single particle motion with core vibrations has been further investigated as correlations always in the ${ }^{11}$ Be case [28], in a similar way as we did. 
Our DCP model is a microscopic version of the quasiparticle-core coupling (QPC) model originally proposed by Bohr and Mottelson [29], and adopted in the past for stable nuclei. The ground state of the even-even nucleus (core) is supposed to be known as the vacuum state for the quasiparticle operators. The wave functions of the oddmass system can be written as

$$
\left|j m, E_{\lambda}\right\rangle=\sum_{n} z_{n j}|n l j m\rangle+\sum_{j^{\prime} J_{c}} z_{j^{\prime} J_{c}}\left|\left(j^{\prime} J_{c}\right) j m\right\rangle,
$$

where $|n l j m\rangle$ is the one-quasiparticle (1QP) state with orbital and total angular momentum $l$ and $j$, parity $\pi_{j}=$ $(-1)^{l}$ and radial quantum number $n \geq 1$. The second term of eq. (1) corresponds to the core excited components (3QP) with a 1QP $j^{\prime}$ and one-particle-one-hole (twoquasiparticle $(2 \mathrm{QP})$ ) core excitations $J_{c}$ coupled to have total angular momentum $j$.

The effective Hamiltonian of the odd-mass system, is separated into $H_{0}$, which is diagonal in the $1 \mathrm{QP}$ and $2 \mathrm{QP}$ states, and the residual interaction $V_{13}$, which couples $1 \mathrm{QP}$ to $3 \mathrm{QP}$ configurations. The residual interaction used is derived from the M3Y-G matrix interaction [30].

The main feature of the DCP model is the derivation of an effective Schrödinger equation for the single-particle part coupled to core excited configurations, leading to an energy dependent contribution in the single-particle selfenergy operator affecting the separation energy and the wave function [31]. The originality of the method consists in expanding the single-particle component of the wave function of the odd-mass nucleus into a set of unperturbed wave function of fixed orbital and total angular momentum $(l, j)$ but different radial quantum number $n$ ("major shell mixing"). It is due to the fact that the nucleon is rescattered by the core, which can have a very large energy. Then, the high-lying core excitations enter off-the-energyshell into the mass operator of low-lying excitations of the odd-mass system. Since the parity of the eigenstate is fixed by $\pi_{j}$, the core excited configurations are restricted by the parity selection rule $\pi_{j}=\pi_{j^{\prime}} \pi_{J_{c}}$. The single particle strengths $|z|^{2}$ are distributed over the whole spectrum, but in the most cases a fraction is found in an interval of several $\mathrm{MeV}$ around the eigenvalue $E_{\lambda}$. The BSEC are directly related to those solutions which lead to a wave function in which one or several core excited states carry the main part of the strength. However, since also the 1QP strength is non-vanishing these states can still be excited in one-step transfer process.

\subsection{Results of the calculations}

In order to give an interpretation of the continuum states populated in the $\left({ }^{7} \mathrm{Li},{ }^{7} \mathrm{Be}\right) \mathrm{CEX}$ reactions at about $8 \mathrm{MeV} / u$ DCP calculations were done for ${ }^{15} \mathrm{C}[32,33]$ and recently also for ${ }^{11} \mathrm{Be}$ [34].

Being a microscopic approach we first describe the ground state of the even-even core ${ }^{10} \mathrm{Be}$ and ${ }^{14} \mathrm{C}$ nuclei by Hartree-Fock-Bogolyubov (HFB) theory. The proton and neutron single-particle energies and wave functions have

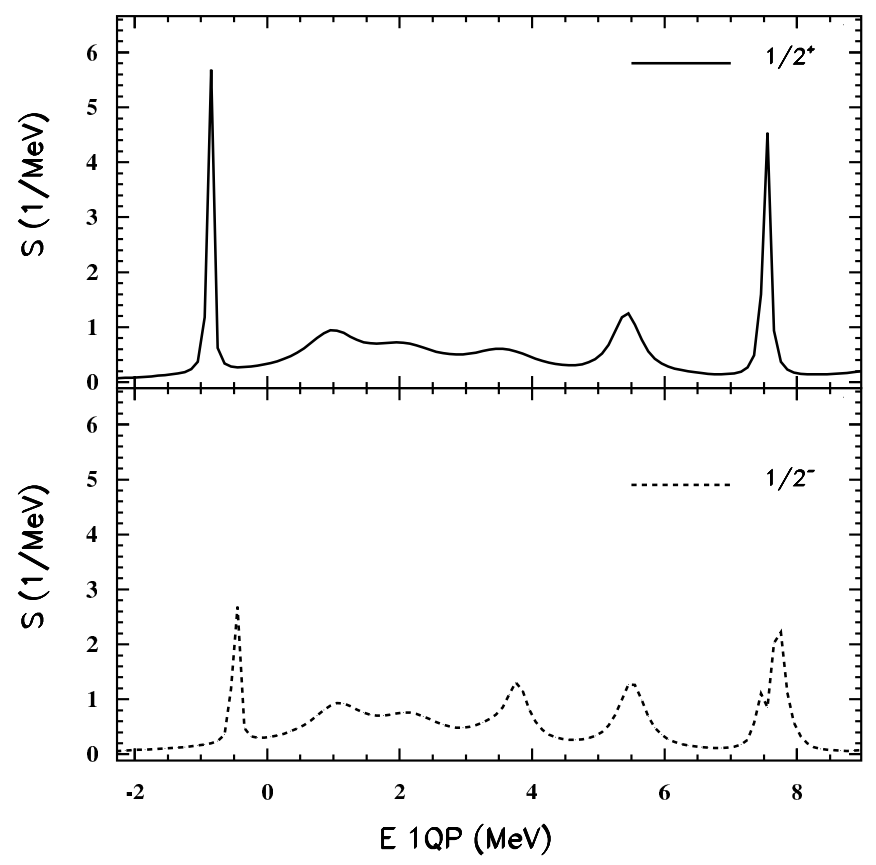

Fig. 3. Calculated single-particle strength of ${ }^{11} \mathrm{Be}$ for $j=1 / 2^{+}$ (solid line) and $j=1 / 2^{-}$(dashed line). See the text for details.

been calculated assuming an isoscalar potential WoodSaxon (WS) shaped, for the central part, and the radial derivative of a WS, for the spin-orbit part. The depth parameters of such potentials have been chosen in order to reproduce the HFB single-particle energies of the core.

Especially the QRPA strength functions of ${ }^{10} \mathrm{Be}\left(2^{+}\right)$ and ${ }^{14} \mathrm{C}\left(1^{-}\right)$are quantitatively well described [34]. The interaction is kept the same for all the studied cases.

In fig. 3 the single-particle strength functions $s_{1 / 2}$ and $p_{1 / 2}$ calculated for the ${ }^{11} \mathrm{Be}$ nucleus are plotted as a function of the 1QP energy. The DCP results of the calculated $s_{1 / 2}$ and $d_{5 / 2}$ single-particle strengths of ${ }^{15} \mathrm{C}$ are presented in fig. 4 . In all the cases, the contribution coming from the natural parity states of the core up to $J_{c}=3$ have been included. Even if the absolute energy values are not quantitatively well reproduced, the level inversion between $2 s_{1 / 2}$ and $1 p_{1 / 2}$ in ${ }^{11} \mathrm{Be}$ is obtained and their energy difference calculated is around $0.390 \mathrm{MeV}$. The energy difference between the $2 s_{1 / 2}$ and $1 d_{5 / 2}$ in ${ }^{15} \mathrm{C}$ is underestimated, being $0.110 \mathrm{MeV}$, but, also here, the experimental level ordering is correctly reproduced. Fragmentation of the strength above $3 \mathrm{MeV}$ and $7 \mathrm{MeV}$ is present in ${ }^{11} \mathrm{Be}$ and ${ }^{15} \mathrm{C}$ calculations, respectively, demonstrating that a detailed microscopic description of the core coupling is very important. Strong overlap between 1QP and 3QP states appears above $8 \mathrm{MeV}$ in the ${ }^{15} \mathrm{C}$ case, with the dominance of $1^{-}, 2^{+}$ and $3^{-14} \mathrm{C}$ exited states. In the ${ }^{11} \mathrm{Be}$ case, contributions of the ${ }^{10} \mathrm{Be}\left(2^{+}\right)$are mainly found at around $3-4 \mathrm{MeV}$. According to our theoretical results, we do not support the necessity to assume in our experimental data configurations where the neutron occupies an orbital with an high orbital angular momentum. 


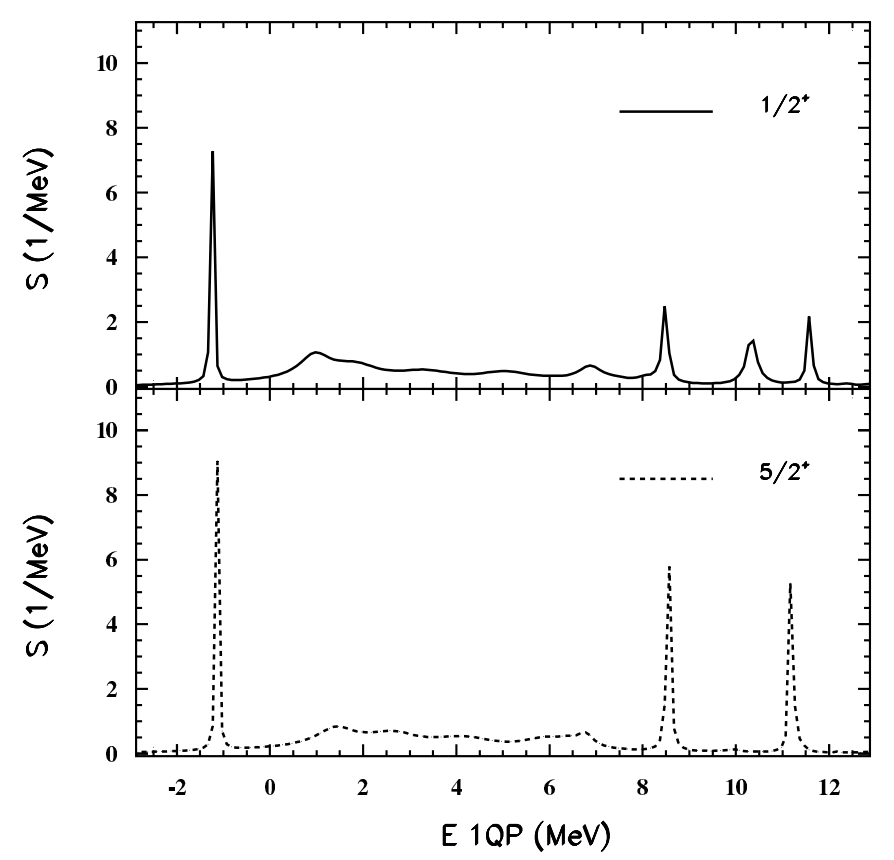

Fig. 4. Calculated single-particle strength of ${ }^{15} \mathrm{C}$ for $j=1 / 2^{+}$ (solid line) and $j=5 / 2^{+}$(dashed line). See the text for details.

Spectroscopic factors in agreement with the values provided experimentally have been obtained. Core excited state configurations like $d_{5 / 2} \otimes{ }^{10} \mathrm{Be}\left(2^{+}\right)$are present in the wave function of the ground state of ${ }^{11} \mathrm{Be}$ at $18 \%$. The main component for the ground state of ${ }^{15} \mathrm{C}$ is represented by the $s_{1 / 2} \otimes{ }^{14} \mathrm{C}\left(0^{+}\right)$configuration.

\section{Other explored $\mathrm{N} \alpha+3 \mathrm{n}$ systems}

In a more general context, the ${ }^{11} \mathrm{Be}$ and ${ }^{15} \mathrm{C}$ nuclei, can be imagined as systems where a hard core made of an integer number of alpha particles $(N \alpha)$ is coupled to three neutrons. Assuming that single-particle excitations of the $\alpha$-clusters are rather unlikely (hard core) at low excitation energy, the few-body dynamics in the nuclear medium can be studied. Since the pairing is expected to play an important role in the three-neutron phase space, a large contribution to it is represented by one valence nucleon coupled to the core, which is made softer by the remaining nucleon pair. Phenomena directly associated to that may be experimentally observed by studying such systems. In this context, experiments involving systems having $N=1$ and $N=4$, i.e. ${ }^{7} \mathrm{He}$ and ${ }^{19} \mathrm{O}$, have already been done.

Concerning ${ }^{7} \mathrm{He}$, the ${ }^{7} \mathrm{Li}\left({ }^{7} \mathrm{Li},{ }^{7} \mathrm{Be}\right){ }^{7} \mathrm{He}$ reaction was performed at about $8 \mathrm{MeV} / u$ using a special target in order to minimized the oxidation of the Li contained in it. The ground state, which is particle instable with respect to the decay into ${ }^{6} \mathrm{He}+\mathrm{n}$ by $0.44 \mathrm{MeV}$ and has a width of $0.160 \mathrm{MeV}$, was easily identified. Another resonant state was found at around $2.90 \mathrm{MeV}$, supposed to be the same populated in other transfer reaction experiments at low and intermediate energies [35]. No trace of the low resonance identified at around $1.2 \mathrm{MeV}$ [36] was found. The

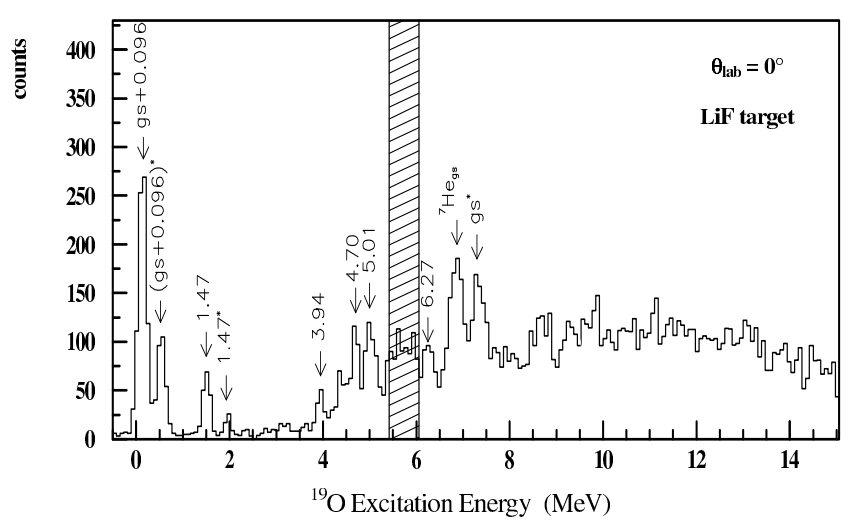

Fig. 5. Excitation energy spectrum at $0^{\circ}$ for the ${ }^{19} \mathrm{~F}\left({ }^{7} \mathrm{Li},{ }^{7} \mathrm{Be}\right){ }^{19}$ reaction at around $8 \mathrm{MeV} / u$. The ground state of ${ }^{7} \mathrm{He}$, which is present due to the Li impurities in the target, is also indicated. The dashed area represents a mask indicating a region not safe for identification due to the background. The states marked with an asterisk are associated to the excitation of ${ }^{7} \mathrm{Be}$ at $0.43 \mathrm{MeV}$.

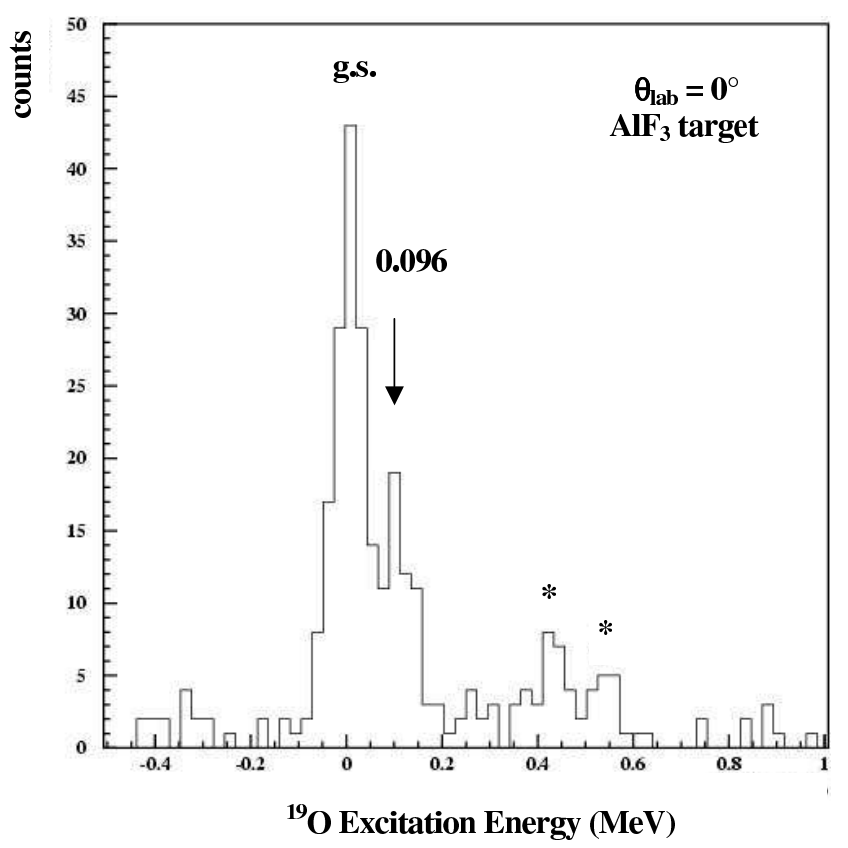

Fig. 6. Detail of the excitation energy spectrum at $0^{\circ}$ obtained for the ${ }^{19} \mathrm{~F}\left({ }^{7} \mathrm{Li},{ }^{7} \mathrm{Be}\right){ }^{19} \mathrm{O}$ reaction at around $8 \mathrm{MeV} / u$ with an $\mathrm{AlF}_{3}$ target. The states marked with an asterisk are associated to the excitation of ${ }^{7} \mathrm{Be}$ at $0.43 \mathrm{MeV}$.

presence of ${ }^{12} \mathrm{C}$ impurities in the target in the $0^{\circ}$ and $4^{\circ}$ spectra did not allow to extract further information.

The ${ }^{19} \mathrm{O}$ nucleus represents an interesting case in order to find similarities with the system having $N=2$ and $N=3$. The populated states in the reaction ${ }^{19} \mathrm{~F}\left({ }^{7} \mathrm{Li},{ }^{7} \mathrm{Be}\right){ }^{19} \mathrm{O}$ were the ground and those at $0.096,1.47$, $3.94,5.01$ and $6.27 \mathrm{MeV}$. In figs. 5 and 6 they are indicated by arrows. The bin size of the $0^{\circ}$ spectrum of fig. 5 is compressed so that the ground and $0.096 \mathrm{MeV}$ states are not resolved. The ground state of the ${ }^{7} \mathrm{He}$, present due to the 
Li impurities of the target, is also indicated. Due to higher neutron separation energy $\left(S_{n}=3.957 \mathrm{MeV}\right)$ in comparison to the ${ }^{11} \mathrm{Be}$ and ${ }^{15} \mathrm{C}$ ones, the interesting region to explore here is at higher excitation energy. Moreover, the presence of the background due to Li and other impurities in the $\mathrm{LiF}$ target, makes it difficult to have clean access to an excitation energy interval greater than $8 \mathrm{MeV}$. The ground and the level at $0.096 \mathrm{MeV}$ of ${ }^{19} \mathrm{O}$ nucleus, are visibly well separated in fig. 6 where a detail of the spectrum measured at $0^{\circ}$, obtained using an $\mathrm{AlF}_{3}$ target, is shown.

\section{Conclusion}

Exploration of excited states in light neutron-rich nuclei is a rich source of information about nuclear structure. We also hope to have indicated a special aspect of nuclear continuum dynamics which might be of relevance for reaction rates and lifetimes of neutron-rich nuclei in astrophysical processes. Excited states in ${ }^{11} \mathrm{Be},{ }^{15} \mathrm{C},{ }^{7} \mathrm{He}$ and ${ }^{19} \mathrm{O}$ have been investigated via the $\left({ }^{7} \mathrm{Li},{ }^{7} \mathrm{Be}\right)$ at very similar bombarding energy $(\sim 8 \mathrm{MeV} / u)$. The obtained high-energy resolution has been crucial for that purpose. A dominant observed feature in the ${ }^{15} \mathrm{C}$ and ${ }^{11} \mathrm{Be}$ cases is the strong fragmentation of strength in BSEC at high excitation energy. The use of refined microscopic theories, such as the DCP model, has been fundamental in order to interpret them as single-particle states where the neutron is coupled to core excited states. The experiments and their analysis discussed here are considered as a first step into the largely unexplored regime of continuum dynamics and BSEC states in weakly bound nuclei. The results deserve further work, especially also in order to clarify the role of such states in astrophysical processes.

Particularly interesting for this phenomenology is the exploration of $N \alpha+3 \mathrm{n}$ like nuclei with $N>4$. They still have not been explored since the cross sections at this low bombarding energies were expected to go down, making the experiments quite difficult. On the other hand, the study of these heavier systems is very important in order to learn about the influence of mass and asymmetry parameters on these phenomena. The advent of the MAGNEX spectrometer [37] at the LNS-INFN in Catania will allow to go on with the systematics by studying less favorable cases, fully exploiting the large solid angle. This device will make it possible to perform measurements with increasing statistics and much less number of angle settings, preserving a good energy resolution.

\section{References}

1. J.S. Winfield et al., Nucl. Phys. A 683, 48 (2001).

2. R. Palit et al., Phys. Rev. C 68, 034318 (2004).

3. G. Baur, H. Lenske, Nucl. Phys. A 282, 201 (1977).

4. H. Fuchs et al., Nucl. Phys. A 343, 133 (1980).

5. F. Cappuzzello et al., Phys. Lett. B 516, 21 (2001).

6. F. Cappuzzello et al., Europhys. Lett. 75(6), 766 (2004).

7. F.J. Eckle et al., Nucl. Phys. A 506, 199 (1990).

8. P. von Neumann-Cosel et al., Nucl. Phys. A 516, 385 (1990).

9. H. Lenske, J. Phys. G 24, 1429 (1998).

10. S.M. Austin et al., Phys. Rev. Lett. 44, 972 (1980).

11. S. Nakayama et al., Phys. Rev. Lett. 67, 1082 (1991).

12. S. Nakayama et al., Phys. Lett. B 246, 342 (1990); Nucl. Phys. A 507, 515 (1990).

13. W. von Oertzen, Nucl. Phys. A 482, 357c (2004).

14. J. Cook et al., Phys. Rev. C 30, 1538 (1984).

15. A. Etchegoyen et al., Phys. Rev. C 38, 2124 (1988).

16. J. Jänecke et al., Phys. Rev. C 54, 1070 (1996).

17. F. Cappuzzello et al., Nucl. Phys. A 739, 30 (2004).

18. W.P. Alford, B.M. Spicer, Advances in Nuclear Physics edited by J.W. Negel, E. Vogt, Vol. 24 (Plenum, New York, 1998).

19. F. Osterfeld, Rev. Mod. Phys. 64, 491 (1992).

20. G. Ohlsen, Nucl. Instrum. Methods 37, 240 (1965).

21. J.D. Garrett et al., Phys. Rev. C 10, 1730 (1974).

22. F.T. Baker et al., Phys. Rep. 289, 235 (2001).

23. S.E.A. Orrigo et al., Proceedings of the 10th International Conference on Nuclear Reaction Mechanism, Villa Monastero, Varenna, 9-13 June 2003, published in Suppl. J. Milan Univ. (Ed. Ricerca Scientifica ed Educazione Permanente, Milano, 2003) p. 147.

24. S.E.A. Orrigo, PhD Thesis, Università di Catania (2004).

25. S.E.A. Orrigo et al., to be published in Phys. Lett. B.

26. G.F. Bertsch et al., Rev. Mod. Phys. 55, 287 (1983).

27. N. Vinh Mau, Nucl. Phys. A 592, 33 (1995).

28. G. Gori et al., Phys. Rev. C 69, 041302(R) (2004).

29. A. Bohr, B. Mottelson, Nuclear Structure, Vols. 1 and 2 (Benjamin, New York, 1969 and 1970).

30. F. Hofmann, H. Lenske, Phys. Rev. C 57, 2281 (1998).

31. H. Lenske, Prog. Part. Nucl. Phys. A 693, 616 (2001).

32. C. Nociforo, PhD Thesis, Università di Catania (2001).

33. C. Nociforo et al., Acta Phys. Pol. B 34, 2387 (2003).

34. C. Nociforo, H. Lenske, to be submitted.

35. F. Ajzenberg-Selove, Nucl. Phys. A 708, 3 (2002).

36. M. Meister et al., Phys. Rev. Lett. 88, 102501 (2002).

37. A. Cunsolo et al., Nucl. Instrum. Methods 484, 56 (2002). 